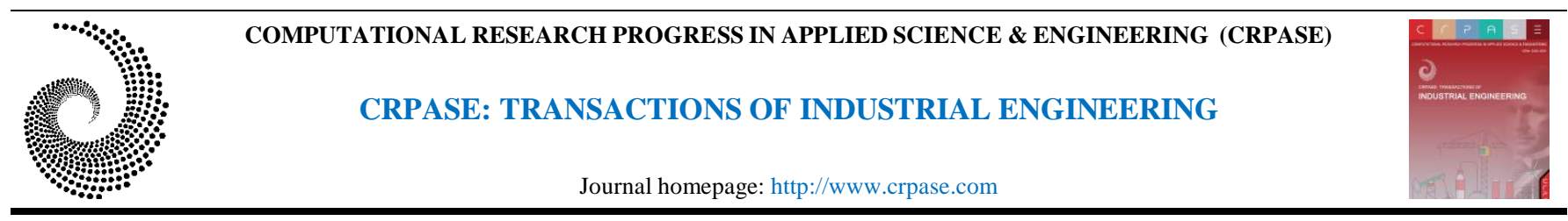

CRPASE: Transactions of Industrial Engineering 7 (3) Article ID: 2392, 1-4, September 2021

ISSN 2423-4591

Review Article

\title{
A Comprehensive Review of Driver's Attention and the Evaluation Methods
}

\author{
Elahe Abbasi, Yueqing Li* \\ Department of Industrial and Systems Engineering, Lamar University, 4400 MLK Blvd., PO Box 10009, Beaumont, Texas 77710, USA
}

\begin{abstract}
Keywords
Driver distraction,

Cognitive distraction,

Vehicle-Based Measures,

Physiological Measures,

Behavioral Measures,

Driving Performance.

Abstract

With the increasing number of people killed by distracted driving, drivers' attention has become a fundamental challenge recently. Performing non-driving-related tasks can make drivers manually, visually, and cognitively distracted. Therefore, there are various definitions and categories for distraction based on the distraction source. Yet, detecting manual and visual distractions are easily but cognitive distractions are hard to identify and measure. Thus, detection, assessment, and evaluation of driver's distraction seem crucial in the safety area. This study aims to (a) present different definitions of driver distraction, (b) introduce various methods to detect driver inattention, and (c) discuss each method's pros and cons and give an insight for an all-inclusive measurement to detect drivers' distraction.
\end{abstract}

\section{Introduction}

As reported by National Highway Traffic Safety Administration (NHTSA), one of the leading causes of traffic crashes is distracted driving. Distraction-affected crashes included 8 percent of fatal crashes and 15 percent of injury crashes in 2018 [1]. Many factors can affect drivers' attention, like using a cellphone, interacting with In-vehicle Information Systems (IVIS), applying music player, eating, drinking, adjusting the hair, checking the makeup, and even daydreaming or just becoming lost in thought. Hence, regarding the tasks drivers perform, they can become manually, visually and mentally (cognitively) distracted. Visual and manual distractions can easily and directly be detected and measured. But cognitive distraction is hard to detect and evaluate directly. Therefore, other approaches have been utilized to measure it. Accordingly, there are several definitions and techniques to evaluate and measure drivers' attention. A brief review of these approaches is presented in this study, focusing on different definitions of distraction and various methods of measuring it based on the distraction type.

\section{Driver Distraction Definitions and Categories}

The term distraction has many faces and several aspects and can affect drivers in various ways. Thus, there is no generally accepted definition for it with the existing knowledge. Over past years, more than 50 definitions have been found for driver's distraction and inattention [2] which each has its concentrations, gaps, pros, and cons. Basically, regarding the area of interest of the researchers, different definitions can be utilized. For example, the scientist may focus on drivers' inattention and define distraction as "insufficient, or no attention, to activities critical for safe driving" [3]. Some other studies concentrate on the driver's diversion of attention which goes to the "divert driver attention away from the driving task to focus on another activity" [1,4,5]. Moreover, some other cases pay more attention to the delay in showing an appropriate reaction to other subjects while performing the driving task. Thus, they apply "delayed in the recognition of information needed to safely accomplish the driving task because some event, activity, object, or person within or outside the vehicle compels or induces the driver's shifting attention away from the driving task" as a definition for distraction $[6,7]$.

\footnotetext{
* Corresponding Author: Yueqing Li

E-mail address: yueqing.li@lamar.edu -Tel, (+1) 409-880-7500
}

Received: 24 August 2021; Revised: 9 September 2021; Accepted: 11 September 2021 https://doi.org/10.52547/crpase.7.3.2392 
As stated by NHTSA, driver distraction can generally be divided into three major categories: visual, manual, and cognitive distraction [8]. Visual distraction happens when drivers look away from the road to achieve some information or control a device, for instance, choosing a song, checking a text message, or answering a call. Manual distraction occurs when drivers remove their hands off the wheel, for example, trying to reach back seats, eating, drinking, adjusting the hair, or checking the makeup. Cognitive distraction takes place when drivers think about something unrelated to their vehicle control duties like daydreaming, or just becoming lost in thought [8]. Any type of these distractions can have an impact on drivers' performance independently or in combination with other forms of distractions.

\section{Distraction Measurements}

Regardless of the differences between distraction types, both cognitive and visual distractions are regarded as significant factors in determining driver behavior. Visual distraction can be indicated by the number of glances, mean glance duration, single glance duration, percent dwell time, total dwell time, blink duration, pupil diameter, etc [9-11]. Visual distraction can impact drivers' performance in many ways either directly like by diminishing perception, increasing glance duration, or indirectly by increasing the drivers' vigilance. Enhancing the vigilance, motorists need to reduce their speed and increase the distance with the leading car to be able to show appropriate response to any potential unexpected situation [9]. Visual and manual distraction can easily and directly be detected and followed through driver's behaviors like the time they look at the billboards or take their hands off the wheel to play music. However, cognitive distraction is internal and hard to detect and notice directly. Thus, to measure cognitive distraction, most inquiries applied other approaches like driving performance, secondary task performance, physiological measures, subjective assessments, and behavior measures $[12,13]$.

\subsection{Driving Performance (Vehicle-Based Measures)}

One of the major approaches that researchers apply to identify driver's distraction is driving performance which can be distinguished through vehicle-based measures [14]. A bunch of studies in this area demonstrated that performing distracting tasks while driving, drivers showed significant changes in vehicle speed $[15,16]$, reaction time $[17,18]$, missing traffic signs and lane-keeping position [19]. For instance, Engström et al. (2005) applied measurements like vehicle speed deviation, steering wheel angle and lane position to evaluate driver's performance. Their results revealed getting distracted, drivers reduce their speed and increase lane- keeping variation [15]. Furthermore, Ahangari et al. (2020) measured the driver's behavior such as speed, throttle, brake, steering velocity, offset from road center, and lane change under different distraction sources. Their analysis indicated that driver's behavior like lane changing, speed fluctuation, and road center deviation was significantly meaningful when they were driving under distraction conditions [20]. In addition, some studies concluded that the performance of distracted drivers can increase the crash risk by rising the braking frequency and mean reaction times to a stimulus or steering and braking responses [17,21,22].

As stated earlier, driving performance measures relied on drivers' performance. But some drivers may drive without any deviation even when they get distracted. Hence, this method can detect distraction to some extent, and the fusion of this approach with other techniques of evaluating driver's distraction is highly recommended.

\subsection{Secondary Task Performance}

The main task for drivers is driving and, they can get distracted when they are busy concentrating on a secondary task like using a cellphone, music player, or In-vehicle Information Systems (IVIS), etc. Therefore, involving in any kind of non-driving-related secondary task or activity can be considered as a distraction for motorists [13]. This can be also measured by the number of detecting a specific event/object or the number of wrong answers to some specific questions [12].

Yet, performing a secondary task is not necessarily equal to getting distracted. Drivers can perform secondary tasks and do not get distracted because of the low level of distractive activity, drivers multitasking, etc. Therefore, using this method can be useful and efficient in conjunction with other methods.

\subsection{Physiological Measures}

There are driving situations in which the drivers are distracted, but they did not show any distraction in their performance like changing or leaving the lane. Therefore, distraction identification cannot happen just based on driving performance [14]. Hence, to avoid this problem, the physiological measures can be applied. In this method physiological (e.g., neurological, cardiovascular and ocular) measures like heart rate (HR), skin conductance responses (SCRs), blood pressure, respiration, hormone levels, EEG, oxyhemoglobin/ deoxyhemoglobin changes [23] can be used to detect driver's inattention. For example, it is found that by the increment and decrement of the activity level of the cerebral cortex region, the blood flow in this area varies accordingly [24]. This phenomenon leads to an increase in oxygenated hemoglobin $\left(\mathrm{HbO}_{2}\right)$ and a decrease in deoxygenated hemoglobin $(\mathrm{HbR})$ in the active brain area [25]. Heart rate can easily be used to detect the driver's attention as well. Cognitively distracted, the driver's heart rate shows significant changes and increments [14,26].

As the physiological signals are not based on drivers' responses, they can be considered a reliable method to detect the attention of the drivers.

\subsection{Subjective Assessments}

When the drivers' inattention is detected by either the participants, regarding their own opinion [10, 20, 27] or external trained observers $[12,28]$ based on their evaluation of the driver's performance, this method is called subjective assessment [29]. There are some standard questionnaires include NASA task load index (NASA-TLX), driving 
activity load index (DALI), subjective workload assessment technique (SWAT), rating scale mental effort (RSME), and modified Cooper Harper (MCH) scale which is usually applied for self-report distraction and completed by participants immediately after finishing each experiment [12]. In the subjective assessment method by external evaluators, perceptual evaluations from external observers, subjects who did not participate as drivers during the recordings are used to identify distraction. The assumption is that trained external evaluators with driving experience can detect participant's distraction by watching videos showing both the drivers' behavior and the road [29].

In this method, the level of distraction for a specific interval like every 5 minutes is measured. Therefore, sudden distraction changes cannot be taken into consideration. Also, as the assessment is based on the subject's idea, their opinion may not be precise and accurate. As a result, subjective assessment is an appropriate method in combination with other measurements or can be used to confirm inattention through other means.

\subsection{Behavioral Measures}

Vision has been asserted as the largest single resource available to the driver and the primary processing input in driving [14]. Behavioral measures like eye movement patterns and head positions can easily be collected by eye trackers, or by application-specific computer vision techniques [13,30,31,32] Various measures from eye movement matrices like eye blinks, fixation, gaze angle, pupil dilation, and glance duration have been used to find distraction $[14,29,33,34]$. It was found that distracted driving is in association with the high frequency of off-road glances, longer total eye-off-road time [17], high frequency and duration increment of eye blink, and dilated pupil $[35,36]$.

A gaze behavior is determined as a fixated gaze on an area in the targeting environment or a shift in gaze from one environmental area to another [37]. Gaze angle and gaze duration are applied as measures to figure out if the drivers concentrate on their driving tasks or they take more time performing other tasks like interacting with IVIS [14]. There are studies that reveal distracted drivers show a significant decrease in their glancing at the instrument panel and side mirrors [14,22]. Moreover, when drivers were distracted visually and cognitively, the standard deviation of gaze angle was decreased substantially [15], and longer interactions occurred for some tasks [38].

Fixations are the moments where a respondent's gazepoint remains almost stationary for a minimum amount of time [29]. It is thought that, for the brain to meaningfully process the visual information, our eyes need to remain in one location for a minimum amount of time (about 100 milliseconds) [39]. In that sense, fixations could be understood as the moments that respondents meaningfully look at something. The fixation position and duration may be related to attention direction and the amount of information perceived from the focused region [17]. Several studies have shown links between fixation duration, gaze angle and distraction [9,29]. For instance, it has been indicated that doing secondary tasks, like interacting with IVIS, increment in the fixations in the center of the roadway and decrement in the fluctuation of fixation position is expected [38].

The pupil is the part of the iris that allows light to pass through to the retina [14]. It was found that mental effort and cognitive distraction can affect pupil size [35].

Although this method has some advantages and is used by lots of researchers it has some disadvantages too. The outputs of this method are mainly extracted from eye information. Yet, eye information is in a strong relationship with other variables, like, the changes in light, vibration, noise, etc. which may affect the output data. For instance, pupil diameter is highly sensitive to light changes that are not necessarily related to the driver's inattention [40].

\section{Discussion}

As previously mentioned, there are various definitions and techniques to identify distraction while most of them have their strength and weakness. To develop a comprehensive system to detect driver's attention, applying a combination of measurements is needed. To this aim, some studies fuse various methods to identify distraction. For instance, the combination of cognitive and visual distraction has been investigated frequently $[4,15,23]$. But there are very limited studies that explore all sources of distraction or utilize its different features simultaneously. However, regarding technological advancement, performing allinclusive distraction studies by modern equipment (e.g., functional Near-Infrared Spectroscopy (fNIRS), modern eye tracker and driving simulators) is needed. These researches expected to consider various driving features like driving performance, physiological, behavioral, and subjective measures simultaneously to increase the safety [41] and reduce crashes [42].

\section{Conclusions}

Distracted driver behaviors can vary regarding the source of distraction. They may get distracted either manually, visually, cognitively, or the combination of all these types. Moreover, there are various methods to detect driver inattention and measure it. While each technique has its own merits and demerits, the combination of numerous ways for detecting inattention will pave the way for an efficient detection system.

\section{References}

[1] National Center for Statistics and Analysis, Distracted driving 2018 (Research Note. Report No. DOT HS 812 926), Washington, DC: National Highway Traffic Safety Administration, April 2020.

[2] K. Kircher, C. Ahlstrom, Minimum Required Attention: A HumanCentered Approach to Driver Inattention, Human Factors 59 (2017) 471- 484.

[3] M. L. Cunningham, M. Regan, Driver Distraction and Inattention in the Realm of Automated Driving, IET Intelligent Transport Systems (2017) 1-7.

[4] D. L. Strayer, Cooper, J. M. Goethe, R. M., D. J. Getty, F. Biondi, Visual and Cognitive Demands of Using In-Vehicle Information Systems, Washington, D.C., AAA Foundation for Traffic Safety 2017.

[5] J. Hedlund, H. Simpson, D. Mayhew, Summary of proceedings and recommendations, International Conference on Distracted Driving. Torento, Traffic Injury Research Foundation and Canadian Automobile Association 2006. 
[6] A. Aksjonov, P. Nedoma, V. Vodovozov, E. Petlenkov, A Method for Detection and Evaluation of Driver Distraction Induced by InVehicle Information Systems. IECON - 44th Annual Conference of the IEEE Industrial Electronics Society, Washington, DC: IEEE, 2018.

[7] J. C. Stutts, D. W. Reinfurt, L. Staplin, C. Hill, The Role of Driver Distractionin Traffic Crashes, Washington, DC: AAA Foundation for Traffic Safety 2001.

[8] National Center for Statistics and Analysis, Overview of the National Highway Traffic Safety Administration's Driver Distraction Program (Research Note. Report No. DOT HS 811 299), Washington, DC: National Highway Traffic Safety Administration, April 2010.

[9] J. Ma, Z. Gong, J. Tan, Q. Zhang, Y. Zuo, Assessing the driving distraction effect of vehicle HMI displays using data mining techniques, Transportation Research Part F 69 (2020) 235-250.

[10] A.K. Kraft, F. Naujoks, J. Wörle, A. Neukum, The impact of an in-vehicle display on glance distribution in partially automated driving in an on-road experiment, Transportation Research Part F 52 (2018) 40-50.

[11] Q. Wu, An Overview of Driving Distraction Measure Methods, IEEE 10th International Conference on Computer-Aided Industrial Design \& Conceptual Design, Wenzhou, China: IEEE, (2009) 2391-2394.

[12] N. Li, C. Busso, Predicting Perceived Visual and Cognitive Distractions of Drivers With Multimodal Features, IEEE Transactions on Intelligent Transportation Systems 16 (2016) 5165 .

[13] L. Tianchi, Y. Yang, G.B. Huang, Y. K. Yeo, Z. Lin, Driver Distraction Detection Using Semi-Supervised Machine Learning, IEEE Transactions on Intelligent Transportation Systems 17 (2016) $1108-1120$.

[14] S. Arun, K. Sundaraj, M. Murugappan, Driver Inattention Detection Methods : A Review, IEEE Conference on Sustainable Utilization and Development in Engineering and Technology, Kuala Lumpur, Malaysia, (2012) 1-6.

[15] J. Engström, E. Johansson, J. östlund, Effects of visual and cognitive load in real and simulated motorway driving, Transportation Research Part F 8 (2005) 97-120.

[16] S. G. Charlton, Driving while conversing: Cell phones that distract and passengers who react, Accident Analysis and Prevention 41 (2009) 160-173.

[17] Y. Liang, Detecting driver distraction, USA: University of Iowa (Thesis), (2009)

[18] F. Tango, \& M. Botta, Evaluation of Distraction in a DriverVehicle-Environment Framework: An Application of Different Data-Mining Techniques, 9th Industrial Conference, ICDM 2009, Leipzig, Germany, (2009) 176-190.

[19] S. Ahangari, M. Jeihani, A. Dehzangi, A Machine Learning Distracted Driving Prediction Model, 3rd International Conference on Vision, Image and Signal Processing, Vancouver BC Canada: Association for Computing Machinery, New York, NY, United States. (2019)1-6.

[20] S. Ahangari, M. Jeihani, B. Salahshour, M. Ndegwa, A Comprehensive Analysis of Distracted Driving Using a Driving Simulator, International Journal for Traffic and Transport Engineering 10 (2020) 252-265.

[21] C. Neubauer, G. Matthews, Fatigue and voluntary utilization of automation in simulated driving, Human Factors 54 (2012) 734 746.

[22] R. Ramnath, N. Kinnear, S. Chowdhury, T. Hyatt, Interacting with Android Auto and Apple CarPlay when driving: The effect on driver performance, TRL Limited (TRL), 2020.

[23] Y. Yang, The effect of increased workload on driving performance and visual behaviour, Southampton, England: University of Southampton (Doctoral thesis), 2011.

[24] Z. Liu, M. Zhang, G. Xu, C. Huo, Q. Tan, Z. Li, Q. Yuan, Effective Connectivity Analysis of the Brain Network in Drivers during Actual Driving Using Near-Infrared Spectroscopy, Frontiers in Behavioral Neuroscience 11 (2017).

[25] C.T. Lin, J.T. King, C.H. Chuang, W. Ding, W.Y. Chuang, L.D. Liao, Y.K. Wang, Exploring the Brain Responses to Driving
Fatigue Through Simultaneous EEG and fNIRS Measurements, International Journal of Neural Systems (2019) 1-12.

[26] E. Pakdamanian, S. Sheng, S. Baee, S. Heo, S. Kraus, L. Feng, DeepTake: Prediction of Driver Takeover Behavior using Multimodal Data, CHI Conference on Human Factors in Computing Systems, Yokohama, Japan: ACM, New York, NY, USA, 2021.

[27] S. Pourfalatoun, E. E. Miller, User perceptions of automated Truck-Mounted attenuators: Implications on work zone safety, Traffic Injury Prevention 22 (2021) 413-418.

[28] J. J. Jain, C. Busso, Assessment of driver's distraction using perceptual evaluations, self assessments and multimodal feature analysis, 5th Biennial Workshop on DSP for In-Vehicle Systems, Kiel, Germany, (2011)1-8.

[29] L. Tianchi, Clustering and semi supervised classification with application to driver distraction detection, Singapore: Nanyang Technological University (Doctoral thesis), 2018.

[30] M. Ebnali, R. Fathi, R. Lamb, S. Pourfalatoun, S. Motamedi, Using Augmented Holographic UIs to Communicate Automation Reliability in Partially Automated Driving, Workshop proceedings Automation Experience across Domains In conjunction with CHI, Honolulu, HI, USA, (2020) 1-8.

[31] A. S. Le, H. Aoki, F. Murase, K. Ishida, A Novel Method for Classifying Driver Mental Workload Under Naturalistic Conditions With Information From Near-Infrared Spectroscopy, Frontiers in Human Neuroscience 12 (2018) 1-12.

[32] E. Pakdamanian, L. Feng, I. Kim, The Effect of Whole-Body Haptic Feedback on Driver's Perception in Negotiating a Curve, Proceedings of the Human Factors and Ergonomics Society 2018 Annual Meeting, Philadelphia, PA, USA, (2018) 19-23.

[33] Z. Gong, J. Ma, Vehicle central display layout evaluation based on driver distraction simulator test, JSAE Annual Congress (Spring), Yokohama, Japan, May (2018) 23-25.

[34] B. Donmez, L. N. Boyle, J. D. Lee, Safety implications of providing real-time feedback to distracted drivers, Accident Analysis and Prevention 39 (2007) 581-590.

[35] S. Benedetto, M. Pedrotti, L. Minin, T. Baccino, A. Re, R. Montanari, Driver workload and eyeblink duration, Transportation Research Part F 14 (2011) 199-208.

[36] N. Merat, A. H. Jamson, F. C. Lai, O. Carsten, Highly Automated Driving, Secondary Task Performance, and Driver State, Human Factors and Automation in Vehicles, October, (2012) 762-771.

[37] J. N. Vickers, Gaze Control in Basketball Foul Shooting, Studies in Visual Information Processing 6 (1995) 527-541.

[38] M. L. Reyes, J. D. Lee, Effects of cognitive load presence and duration on driver eye movements and event detection performance, Transportation Research Part F 11 (2008) 391-402.

[39] imotions, imotion help center, (2018). Available online: https://help.imotions.com/hc/en-us/articles/360015276380Export-AOI-metrics-per-respondent-iMotions-8-2-and-earlier-

[40] A. S. Le, T. Suzuki, H. Aoki, Evaluating driver cognitive distraction by eye tracking: From simulator to driving, Transportation Research Interdisciplinary Perspectives 4 (2020) 1 7.

[41] M. J. Samet, Development of Accident Modification Factors in Two-Lane Highways, Computational Research Progress in Applied Science \& Engineering 2 (2016) 168-172.

[42] S. M. Hosseinian, V. Najafi Moghaddam Gilani, H. Tahmasbi Amoli, M Nikookar, A. Orouei, Presentation of Analytical Methods for Better Decision Making about the Most Important Factor Influencing Rural Accidents, Mathematical Problems in Engineering (2021) 1-16. 